\title{
AN INDUCTION THEOREM FOR GROUPS ACTING ON TREES
}

\author{
MARTIN H. WEISSMAN
}

\begin{abstract}
If $G$ is a group acting on a locally finite tree $X$, and $\mathcal{S}$ is a $G$ equivariant sheaf of vector spaces on $X$, then its compactly-supported cohomology is a representation of $G$. Under a finiteness hypothesis, we prove that if $H_{c}^{0}(X, \mathcal{S})$ is an irreducible representation of $G$, then $H_{c}^{0}(X, \mathcal{S})$ arises by induction from a vertex or edge stabilizing subgroup.

If $\mathbf{G}$ is a reductive group over a nonarchimedean local field $F$, then Schneider and Stuhler realize every irreducible supercuspidal representation of $G=$ $\mathbf{G}(F)$ in the degree-zero cohomology of a $G$-equivariant sheaf on its reduced Bruhat-Tits building $X$. When the derived subgroup of $\mathbf{G}$ has relative rank one, $X$ is a tree. An immediate consequence is that every such irreducible supercuspidal representation arises by induction from a compact-mod-center open subgroup.
\end{abstract}

\section{INTRODUCTION}

According to a folklore conjecture, every irreducible supercuspidal representation of a reductive $p$-adic group arises by induction from a compact-mod-center open subgroup. This is proven for $G L_{n}$ by Bushnell-Kutzko [BK93, for many classical groups by Stevens [Ste08, and for tame supercuspidals by Ju-Lee Kim Kim07. (exhaustion) and Jiu-Kang Yu Yu01 (construction). Outside of $G L_{n}$, these results require some assumptions on characteristic and residue characteristic.

Here we prove the conjecture for all groups of relative rank one-those whose Bruhat-Tits building is a tree. Our method is less constructive, but follows directly from results of Schneider-Stuhler [SS97] and the geometry of equivariant sheaves on trees. No restrictions on residue characteristic (or characteristic!) are required, so the result seems new in many cases, e.g., for $S U_{3}$ in residue characteristic two and for quaternionic unitary groups.

There are nine classes of groups of relative rank one over a nonarchimedean local field $F$, if one uses the Tits index to organize them [Tit79, §4]. These are conveniently tabulated and described in notes of Carbone Car. Their simplyconnected forms are $S L_{2}(F)$ and $S L_{2}(D)$ (for $D$ a division algebra of any degree over $F)$, the quasisplit unitary groups $S U_{3}^{E}$ and $S U_{4}^{E}(E / F$ a separable quadratic field extension), and five types of quaternionic unitary groups $S U_{2}(D, s), S U_{3}(D, s)$, $S U_{3}(D, h), S U_{4}(D, h), S U_{5}(D, h)$ which have absolute types $\mathrm{C}_{2}, \mathrm{C}_{3}, \mathrm{D}_{3}, \mathrm{D}_{4}$, and $\mathrm{D}_{5}$, respectively. For these, $D$ denotes a quaternion division algebra over $F, h$ a nondegenerate Hermitian form, and $s$ a nondegenerate skew-hermitian form. Previous results have addressed groups in three of these nine classes in a nonuniform

Received by the editors October 30, 2018, and, in revised form, December 10, 2018.

2010 Mathematics Subject Classification. Primary 20G25, 20E08, 22E50.

The Simons Foundation Collaboration Grant \#426453 supported this work. 
manner, typically under restrictions on isogeny class, characteristic, and residue characteristic.

Main results. The main result of the paper is Theorem 2.4, described here.

Theorem. Let $\mathcal{S}$ be a $G$-equivariant sheaf on a locally finite tree $X$. Suppose that $\mathcal{S}$ has finite 0-rank (see \$2.5). If $H_{c}^{0}(X, \mathcal{S})$ is an irreducible representation of $G$, then $H_{c}^{0}(X, \mathcal{S})$ is isomorphic to a representation of $G$ induced from the stabilizer of a vertex or edge.

Now let $\mathbf{G}$ be a reductive group over a nonarchimedean local field $F$, whose derived subgroup has relative rank one. Let $G=\mathbf{G}(F)$ and let $(\pi, V)$ be an irreducible supercuspidal representation of $G$. The Bruhat-Tits building of $G$ is a locally finite tree $X$. In [SS97, Schneider and Stuhler prove that $(\pi, V)$ arises as $H_{c}^{0}(X, \mathcal{S})$ for a suitable $G$-equivariant sheaf on $X$. This sheaf has finite 0-rank, by the admissibility of smooth irreducible representations. Stabilizers of vertices and edges are compact-mod-center open subgroups of $G$. Hence the theorem above implies the result below.

Corollary. $(\pi, V)$ is isomorphic to a representation of $G$ induced from a compactmod-center open subgroup.

In Ol'77, Ol'sanskiu proves a similar result for the full automorphism group of a Bruhat-Tits tree $X$. The automorphism group $\operatorname{Aut}(X)$ is a much larger group than $G$, but some of the representation theory of $p$-adic groups can be adapted to $\operatorname{Aut}(X)$. Irreducible representations of $\operatorname{Aut}(X)$ are classified as spherical, special, or cuspidal, and $\mathrm{Ol}^{\prime}$ shanskiı proves Ol'77, Theorem 1] that cuspidal representations are induced from compact open subgroups. But this result does not prove that supercuspidal representations of $G$ are induced from compact open subgroups, nor does Ol'shanskii's proof seem to adapt to the representation theory of $G$.

The proof of the main result relies on an inductive argument, whittling down a sheaf $\mathcal{S}$ as much as possible while tracking its cohomology. The key idea is that a sheaf $\mathcal{S}$ on a tree has an elliptic subsheaf $\mathcal{S}^{\text {ell }}$ supported on the zero-skeleton, and (when $\mathcal{S}^{\text {ell }}=0$ ) a unifacial subsheaf $\mathcal{S}^{\text {uni }}$ in which sections propagate only in one direction from each vertex. The cohomology of elliptic and unifacial sheaves are easily computed, and relate to representations induced from vertex and edge stabilizers, respectively. By pulling out elliptic and unifacial subsheaves, repeatedly if necessary, we can relate the cohomology of $\mathcal{S}$ to the cohomology of such subsheaves.

The first part of the article reviews the theory of equivariant sheaves on trees, and describes these elliptic and unifacial subsheaves. The inductive argument for Theorem 2.4 proceeds immediately after. After reviewing the relevant results of Schneider and Stuhler [SS97, we discuss the implications for supercuspidal representations at the end.

\section{SHEAVES ON TREES}

Let $X$ be a locally finite tree with vertex set $V$ and edge set $E$. If $v \in V$ and $e \in E$, then we write $v<e$ to mean that $v$ is an endpoint of $e$. Fix a field $k$. A sheaf on $X$ will mean a cellular sheaf of $k$-vector spaces on $X$. Such a sheaf consists of $k$-vector spaces $\mathcal{S}_{v}$ for every vertex $v \in V$, and $\mathcal{S}_{e}$ for every edge $e \in E$, and linear maps

$$
\gamma_{v, e}: \mathcal{S}_{v} \rightarrow \mathcal{S}_{e}
$$


for all $v<e$. The maps $\gamma_{v, e}$ are called restriction maps and the spaces $\mathcal{S}_{v}, \mathcal{S}_{e}$ are called the stalks of $\mathcal{S}$. We write $(\mathcal{S}, \gamma)$ or sometimes just $\mathcal{S}$ for such a sheaf.

Let $G$ be a group acting on $X$. A $G$-equivariant structure on a sheaf $(\mathcal{S}, \gamma)$ consists of linear maps

$$
\eta_{g, v}: \mathcal{S}_{v} \rightarrow \mathcal{S}_{g v}, \quad \eta_{g, e}: \mathcal{S}_{e} \rightarrow \mathcal{S}_{g e}
$$

for all $g \in G, v \in V, e \in E$, satisfying the following axioms:

- For all $v \in V, e \in E$, the linear maps $\eta_{1, v}$ and $\eta_{1, e}$ are the identity.

- For all $g, h \in G, v \in V$, and $e \in E, \eta_{g, h v} \circ \eta_{h, v}=\eta_{g h, v}$ and $\eta_{g, h e} \circ \eta_{h, e}=$ $\eta_{g h, e}$.

- For all $g \in G$, and $v<e$, we have $\gamma_{g v, g e} \circ \eta_{g, v}=\eta_{g, e} \circ \gamma_{v, e}$.

A $G$-equivariant sheaf on $X$ will mean a sheaf $(\mathcal{S}, \gamma)$ endowed with a $G$-equivariant structure.

2.1. Cohomology. For convenience, fix an orientation on every edge $e \in E$. This orders the endpoints of every edge $e$, and we write $x_{e}, y_{e}$ for the first and second endpoint, respectively. If $v<e$, then write or $(v, e)=1$ if $v=x_{e}$ and $\operatorname{or}(v, e)=-1$ if $v=y_{e}$.

Fix a sheaf $(\mathcal{S}, \gamma)$ on $X$ in what follows. If $v \in V$, and $s \in \mathcal{S}_{v}$, define

$$
\mathrm{d} s=\sum_{e>v} \operatorname{or}(v, e) \cdot \gamma_{v, e}(s) \in \bigoplus_{e>v} \mathcal{S}_{e} .
$$

Note that local finiteness is necessary here, i.e., every vertex is incident to a finite number of edges. The compactly-supported cohomology of $\mathcal{S}$ is then computed by the complex

$$
0 \rightarrow \bigoplus_{v \in V} \mathcal{S}_{v} \stackrel{\mathrm{d}}{\rightarrow} \bigoplus_{e \in E} \mathcal{S}_{e} \rightarrow 0
$$

With reference to the complex above,

$$
H_{c}^{0}(X, \mathcal{S})=\operatorname{Ker} \mathrm{d}, \quad H_{c}^{1}(X, \mathcal{S})=\operatorname{Cok} \mathrm{d} .
$$

If $\mathcal{S}$ is a $G$-equivariant sheaf, then the complex above and its cohomology inherit actions of $G$. Explicitly, if $g \in G$ and $v \in V$, then $g$ sends the summand $\mathcal{S}_{v}$ to the summand $\mathcal{S}_{g v}$ by $\eta_{g, v}$. For an edge $e \in E, g$ sends the summand $\mathcal{S}_{e}$ to the summand $\mathcal{S}_{g e}$ by $\pm \eta_{g, e}$, where the sign depends on whether $\left(x_{g e}, y_{g e}\right)=\left(g x_{e}, g y_{e}\right)$ or $\left(x_{g e}, y_{g e}\right)=\left(g y_{e}, g x_{e}\right)$. In this way, $H_{c}^{i}(X, \mathcal{S})$ is a representation of $G$ on a $k$-vector space for $i=0,1$.

2.2. The elliptic subsheaf. Fix a $G$-equivariant sheaf $(\mathcal{S}, \gamma, \eta)$ on $X$ in what follows. If $v \in V$ and $s \in \mathcal{S}_{v}$, we say that $s$ is elliptic if $\mathrm{d} s=0$, i.e., if

$$
\gamma_{v, e}(s)=0 \text { for all } e>v \text {. }
$$

The elliptic elements of $\mathcal{S}_{v}$ form a subspace $\mathcal{S}_{v}^{\text {ell }} \subset \mathcal{S}_{v}$. If $e$ is an edge, define $\mathcal{S}_{e}^{\text {ell }}=0$. This defines a $G$-equivariant subsheaf of $\mathcal{S}$, which we call the elliptic subsheaf

$$
\mathcal{S}^{\text {ell }} \subset \mathcal{S} \text {. }
$$

By construction, we have

$$
H_{c}^{0}\left(X, \mathcal{S}^{\mathrm{ell}}\right)=\bigoplus_{v \in V} \mathcal{S}_{v}^{\mathrm{ell}} \text { and } H_{c}^{1}\left(X, \mathcal{S}^{\mathrm{ell}}\right)=0
$$


For $x \in V$, let $G_{x}$ denote its stabilizer and $G \cdot x$ its orbit. Then $\mathcal{S}_{x}$ is naturally (via $\eta$ ) a representation of $G_{x}$, and $\mathcal{S}_{x}^{\text {ell }}$ is a $G_{x}$-subrepresentation. Algebraic induction gives a natural identification of $G$-representations,

$$
\bigoplus_{v \in G \cdot x} \mathcal{S}_{v}^{\mathrm{ell}} \equiv \operatorname{cInd}_{G_{x}}^{G} \mathcal{S}_{x}^{\mathrm{ell}}
$$

Above and in what follows, if $K$ is a subgroup of $G$ and $(\sigma, S)$ is a representation of $K, \operatorname{cnd}_{K}^{G}(S)$ denotes "algebraic induction", i.e., the space of functions $f: G \rightarrow S$ supported on a finite number of left $K$-cosets, satisfying $f(g k)=\sigma(k)^{-1} f(g)$ for all $g \in G, k \in K$.

It follows that $H_{c}^{0}\left(X, \mathcal{S}^{\text {ell }}\right)$ is a direct sum of such induced representations:

$$
H_{c}^{0}\left(X, \mathcal{S}^{\mathrm{ell}}\right) \equiv \bigoplus_{G \cdot x \in G \backslash V} \operatorname{cInd}_{G_{x}}^{G} \mathcal{S}_{x}^{\text {ell }} .
$$

2.3. The unifacial subsheaf. Suppose now that $\mathcal{S}^{\text {ell }}=0$. If $v \in V$ and $s \in \mathcal{S}_{v}$, we say that $s$ is unifacial if there exists a unique edge $e>v$ such that $\gamma_{v, e}(s) \neq 0$. Define

$$
\mathcal{S}_{v, e}^{\text {uni }}=\operatorname{Span}_{k}\left\{s \in \mathcal{S}_{v}: s \text { is unifacial and } \gamma_{v, e}(s) \neq 0\right\} .
$$

Since we assume $\mathcal{S}^{\text {ell }}=0$, we find that

$$
\mathcal{S}_{v, e}^{\text {uni }}=\left\{s \in \mathcal{S}_{v}: s \text { is unifacial and } \gamma_{v, e}(s) \neq 0\right\} \sqcup\{0\} .
$$

The spaces $\mathcal{S}_{v, e}^{\text {uni }}$, for various $e$, are linearly independent in $\mathcal{S}_{v}$. Hence putting these spaces together for various edges, we define

$$
\mathcal{S}_{v}^{\text {uni }}=\operatorname{Span}_{k}\left\{s \in \mathcal{S}_{v}: s \text { is unifacial }\right\}=\bigoplus_{e>v} \mathcal{S}_{v, e}^{\text {uni }} .
$$

If $e$ is an edge with endpoints $x, y$, define

$$
\mathcal{S}_{e}^{\text {uni }}=\gamma_{x, e}\left(\mathcal{S}_{x, e}^{\text {uni }}\right)+\gamma_{y, e}\left(\mathcal{S}_{y, e}^{\text {uni }}\right) \subset \mathcal{S}_{e} .
$$

The spaces $\mathcal{S}_{v}^{\text {uni }}$ and $\mathcal{S}_{e}^{\text {uni }}$ define a $G$-equivariant subsheaf $\mathcal{S}^{\text {uni }} \subset \mathcal{S}$, whose cohomology can be described explicitly.

Proposition 2.1. $H_{c}^{1}\left(X, \mathcal{S}^{\text {uni }}\right)=0$.

Proof. We demonstrate that $\mathrm{d}$ is surjective as follows. Suppose $e \in E$ and $s \in \mathcal{S}_{e}^{\text {uni }}$. Write $x=x_{e}$ and $y=y_{e}$. Then there exists $a \in \mathcal{S}_{x, e}^{\text {uni }}$ and $b \in \mathcal{S}_{y, e}^{\text {uni }}$ such that

$$
s=\gamma_{x, e}(a)+\gamma_{y, e}(b) .
$$

We assume that $a=0$ if $\gamma_{x, e}(a)=0$, and $b=0$ if $\gamma_{y, e}(b)=0$. Since $\gamma_{x, e^{\prime}}(a)=0$ and $\gamma_{y, e^{\prime}}(b)=0$ for all $e^{\prime} \neq e$, we find that $s=\mathrm{d}(a-b)$. Hence $\mathrm{d}$ is surjective and $H_{c}^{1}\left(X, \mathcal{S}^{\text {uni }}\right)$ vanishes.

Proposition 2.2. $H_{c}^{0}\left(X, \mathcal{S}^{\text {uni }}\right)=\bigoplus_{e \in E}\left(\gamma_{x_{e}, e}\left(\mathcal{S}_{x_{e}, e}^{\text {uni }}\right) \cap \gamma_{y_{e}, e}\left(\mathcal{S}_{y_{e}, e}^{\text {uni }}\right)\right)$.

Proof. We define auxiliary sheaves $(\mathcal{R}, \rho)$ and $(\mathcal{T}, \tau)$ as follows. For every vertex $v$, define $\mathcal{R}_{v}=\mathcal{S}_{v}^{\text {uni }}$ and $\mathcal{T}_{v}=0$. For every edge $e$, with endpoints $x, y$, define

$$
\mathcal{R}_{e}=\gamma_{x, e}\left(\mathcal{S}_{x, e}^{\text {uni }}\right) \oplus \gamma_{y, e}\left(\mathcal{S}_{y, e}^{\text {uni }}\right), \quad \mathcal{T}_{e}=\gamma_{x, e}\left(\mathcal{S}_{x, e}^{\text {uni }}\right) \cap \gamma_{y, e}\left(\mathcal{S}_{y, e}^{\text {uni }}\right) .
$$

For $v<e$, define $\tau_{v, e}: \mathcal{T}_{v} \rightarrow \mathcal{T}_{e}$ to be the zero map. Define $\rho_{v, e}: \mathcal{R}_{v} \rightarrow \mathcal{R}_{e}$ by $\rho_{v, e}(s)=\gamma_{v, e}(s)$, where the latter is viewed in the summand $\gamma_{v, e}\left(\mathcal{S}_{v, e}^{\text {uni }}\right)$ of $\mathcal{R}_{e}$. 
There is a natural short exact sequence of sheaves on $X$,

$$
\mathcal{T} \hookrightarrow \mathcal{R} \rightarrow \mathcal{S}^{\text {uni }} .
$$

At vertices, the maps are obvious; for edges, the map $\mathcal{T}_{e} \rightarrow \mathcal{R}_{e}$ sends an element

$$
t \in \gamma_{x, e}\left(\mathcal{S}_{x, e}^{\mathrm{uni}}\right) \cap \gamma_{y, e}\left(\mathcal{S}_{y, e}^{\mathrm{uni}}\right)
$$

to the ordered pair $(t,-t)$. The map from $\mathcal{R}_{e}$ to $\mathcal{S}_{e}^{\text {uni }}$ is addition.

Essentially by construction, $H_{c}^{0}(X, \mathcal{R})=H_{c}^{1}(X, \mathcal{R})=0$. Indeed, we can decompose $\mathcal{R}$ as a product of sheaves,

$$
\mathcal{R}=\prod_{v \in V} \mathcal{R}^{(v)}
$$

where $\mathcal{R}^{(v)}$ is supported on the star-neighborhood of $v: \mathcal{R}_{v}^{(v)}:=\mathcal{R}_{v}=\mathcal{S}_{v}^{\text {uni }}$ and $\mathcal{R}_{e}^{(v)}=\gamma_{v, e}\left(\mathcal{S}_{v, e}^{\text {uni }}\right)$ for all $e>v$. Since $\gamma_{v, e}: \mathcal{S}_{v, e}^{\text {uni }} \rightarrow \gamma_{v, e}\left(\mathcal{S}_{v, e}^{\text {uni }}\right)$ is an isomorphism for all $v<e\left(\right.$ recall $\mathcal{S}^{\text {ell }}=0$ ), we find that

$$
\mathrm{d}: \mathcal{R}_{v}^{(v)} \longrightarrow \bigoplus_{e>v} \mathcal{R}_{e}^{(v)}
$$

is an isomorphism (see (2.3) $)$. Hence $H_{c}^{i}\left(X, \mathcal{R}^{(v)}\right)=0$ for $i=0,1$. The compactlysupported cohomology of $\mathcal{R}$ is the direct sum of these, which vanishes.

From the short exact sequence of sheaves, $\mathcal{T} \hookrightarrow \mathcal{R} \rightarrow \mathcal{S}^{\text {uni }}$, the long exact sequence in cohomology gives an identification,

$$
H_{c}^{0}\left(X, \mathcal{S}^{\text {uni }}\right) \equiv H_{c}^{1}(X, \mathcal{T}) .
$$

Since $\mathcal{T}_{v}=0$ for all vertices $v$, we find that

$$
H_{c}^{1}(X, \mathcal{T})=\bigoplus_{e \in E} \mathcal{T}_{e}=\bigoplus_{e \in E}\left(\gamma_{x_{e}, e}\left(\mathcal{S}_{x_{e}, e}^{\text {uni }}\right) \cap \gamma_{y_{e}, e}\left(\mathcal{S}_{y_{e}, e}^{\text {uni }}\right)\right)
$$

A $G$-equivariant structure on $\mathcal{S}$ transports to $G$-equivariant structures on $\mathcal{T}$ and $\mathcal{R}$. It follows that, for any edge $e \in E$, the space $\mathcal{T}_{e}$ is naturally a representation of the stabilizer $G_{e}$. Therefore, we find an identification of representations of $G$,

$$
H_{c}^{0}\left(X, \mathcal{S}^{\text {uni }}\right) \equiv H_{c}^{1}(X, \mathcal{T}) \equiv \bigoplus_{G \cdot e \in G \backslash E} \operatorname{cInd}_{G_{e}}^{G} \mathcal{T}_{e}
$$

2.4. Multifacial sheaves. Now, suppose that $(\mathcal{S}, \gamma)$ is a sheaf on $X$ and $\mathcal{S}^{\text {ell }}=0$ and $\mathcal{S}^{\text {uni }}=0$. Thus, for every $v \in V$ and every nonzero $s \in \mathcal{S}_{v}$, there exist at least two edges $e, f \in E$ such that $v<e$ and $v<f$ and

$$
\gamma_{v, e}(s) \neq 0 \text { and } \gamma_{v, f}(s) \neq 0 .
$$

We call such a sheaf multifacial.

Proposition 2.3. If $\mathcal{S}$ is a multifacial sheaf, then $H_{c}^{0}(X, \mathcal{S})=0$.

Proof. Suppose that $s=\left(s_{v}: v \in V\right) \in H_{c}^{0}(X, \mathcal{S})$. Assume that $s \neq 0$. Then there exists a nonempty finite set $W \subset V$ of vertices such that $s_{v} \neq 0$ if and only if $v \in W$. Let $\Omega$ be the convex hull of $W$ in the tree $X$. In other words, $\Omega$ is the smallest connected subgraph of $X$ containing every vertex from $W$. In particular, $\Omega$ is a finite tree. Moreover, if $\ell$ is a leaf of $\Omega$, then $\ell \in W$; otherwise one could prune the leaf while maintaining connectedness and containment of $W$. Figure 1 displays the geometric argument we give here. 
Let $\ell$ be a leaf of $\Omega$, so there is at most one edge of $\Omega$ having $\ell$ as an endpoint. Since $\ell \in W$, we have $s_{\ell} \neq 0$. Since $\mathcal{S}$ is multifacial, there exists an edge $e$ such that $\ell<e, e$ does not belong to $\Omega$, and $\gamma_{\ell, e}\left(s_{\ell}\right) \neq 0$. Let $v$ be the other endpoint of $e$. Since $\ell$ was a leaf of $\Omega, v \notin \Omega$.

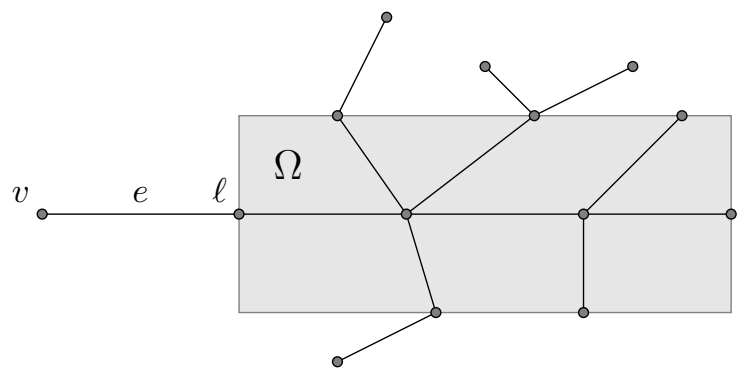

Figure $1 . \ell$ is a leaf of the finite tree $\Omega$ (the subgraph contained in the shaded box), and $e$ is an edge that protrudes outside of $\Omega$.

Since $\mathrm{d} s=0$, we have $(\mathrm{d} s)_{e}=0$. Equivalently,

$$
\gamma_{v, e}\left(s_{v}\right)-\gamma_{\ell, e}\left(s_{\ell}\right)=0 \text {. }
$$

Since $\gamma_{\ell, e}\left(s_{\ell}\right) \neq 0$, this implies $\gamma_{v, e}\left(s_{v}\right) \neq 0$, which implies $s_{v} \neq 0$. But this contradicts the fact that $v \notin \Omega$.

This contradiction proves that $H_{c}^{0}(X, \mathcal{S})=0$.

2.5. The induction theorem. Suppose that $\mathcal{S}$ is a $G$-equivariant sheaf on $X$. We define its 0-rank to be the cardinal number

$$
\operatorname{Rank}^{0}(\mathcal{S})=\sum_{G \cdot v \in G \backslash V} \operatorname{dim}\left(\mathcal{S}_{v}\right) .
$$

For example, if $G \backslash V$ is finite and every stalk of $\mathcal{S}$ is finite-dimensional, then $\operatorname{Rank}^{0}(\mathcal{S})$ will be finite.

Theorem 2.4. Assume that $\operatorname{Rank}^{0}(\mathcal{S})$ is finite. If $H_{c}^{0}(X, \mathcal{S})=0$ or $H_{c}^{0}(X, \mathcal{S})$ is an irreducible representation of $G$, then $H_{c}^{0}(X, \mathcal{S})$ is isomorphic to a representation induced from the stabilizer of a vertex or edge.

Proof. We proceed by induction on $\operatorname{Rank}^{0}(\mathcal{S})$. If $H_{c}^{0}(X, \mathcal{S})=0$, then $H_{c}^{0}(X, \mathcal{S})$ is induced from the zero representation via any subgroup, and the result is trivial. This takes care of the $\operatorname{Rank}^{0}(\mathcal{S})=0$ base case, in particular.

So assume that $\operatorname{Rank}^{0}(\mathcal{S})>0$ and the result has been proven for lower 0-rank. If $H_{c}^{0}(X, \mathcal{S})=0$, then we are done. Otherwise, by Proposition 2.3, we find that $\mathcal{S}^{\text {ell }} \neq 0$ or $\left(\mathcal{S}^{\text {ell }}=0\right.$ and $\left.\mathcal{S}^{\text {uni }} \neq 0\right)$. We consider these two cases below.

If $\mathcal{S}^{\text {ell }} \neq 0$, then $H_{c}^{0}\left(X, \mathcal{S}^{\text {ell }}\right)$ is a nonzero subrepresentation of $H_{c}^{0}(X, \mathcal{S})$. By irreducibility, we find that

$$
H_{c}^{0}(X, \mathcal{S}) \equiv H_{c}^{0}\left(X, \mathcal{S}^{\mathrm{ell}}\right)
$$

By (2.2), this is a direct sum of representations induced from stabilizers of vertices. By irreducibility again, only one $G$-orbit of vertices can support $\mathcal{S}^{\text {ell }}$ and

$$
H_{c}^{0}(X, \mathcal{S}) \equiv \operatorname{cInd}_{G_{x}}^{G} \mathcal{S}_{x}^{\text {ell }}
$$

for some vertex $x \in V$. Thus if $\mathcal{S}^{\text {ell }} \neq 0$, the result holds. 
Next, suppose that $\mathcal{S}^{\text {ell }}=0$ and $\mathcal{S}^{\text {uni }} \neq 0$. Consider the short exact sequence,

$$
\mathcal{S}^{\text {uni }} \hookrightarrow \mathcal{S} \rightarrow \mathcal{S} / \mathcal{S}^{\text {uni }}
$$

Since $\mathcal{S}^{\text {uni }} \neq 0, \mathcal{S}_{v}^{\text {uni }} \neq 0$ for some vertex $v$, and so $\operatorname{Rank}^{0}\left(\mathcal{S} / \mathcal{S}^{\text {uni }}\right)<\operatorname{Rank}^{0}(\mathcal{S})$.

By Proposition 2.1, the long exact sequence in cohomology yields

$$
0 \rightarrow H_{c}^{0}\left(X, \mathcal{S}^{\text {uni }}\right) \rightarrow H_{c}^{0}(X, \mathcal{S}) \rightarrow H_{c}^{0}\left(X, \mathcal{S} / \mathcal{S}^{\text {uni }}\right) \rightarrow 0 .
$$

This is a short exact sequence of $G$-representations, so irreducibility of the middle term yields

$$
H_{c}^{0}(X, \mathcal{S}) \equiv H_{c}^{0}\left(X, \mathcal{S}^{\text {uni }}\right) \text { or } H_{c}^{0}(X, \mathcal{S}) \equiv H_{c}^{0}\left(X, \mathcal{S} / \mathcal{S}^{\text {uni }}\right) .
$$

In the first case, (2.4) and irreducibility yields

$$
H_{c}^{0}(X, \mathcal{S}) \equiv \operatorname{cInd}_{G_{e}}^{G} \mathcal{T}_{e}
$$

for some edge $e \in E$ and the result holds.

In the second case, $H_{c}^{0}(X, \mathcal{S}) \equiv H_{c}^{0}\left(X, \mathcal{S} / \mathcal{S}^{\text {uni }}\right)$. But $\mathcal{S} / \mathcal{S}^{\text {uni }}$ has lower 0-rank. Hence the second case follows from the theorem for sheaves of lower 0-rank.

2.6. Supercuspidal representations. Let $\mathbf{G}$ be a reductive group over a nonarchimedean local field $F$, and let $X$ be the reduced Bruhat-Tits building of $G=$ $\mathbf{G}(F)$. Then $X$ is a cell complex, and $G$ acts on $X$ in such a way that

- there are finitely many $G$-orbits on the set of cells of $X$;

- if $x \in X$, the stabilizer $G_{x}$ is a compact-mod-center open subgroup of $G$.

In SS97, Schneider and Stuhler relate finite-length smooth representations of $G$ to $G$-equivariant sheaves on $X$. We sketch their construction briefly here. Let $(\pi, V)$ be an finite-length smooth representation of $G$ on a complex vector space. For all $x \in X$, Schneider and Stuhler define a filtration of $G_{x}$ by compact open subgroups $G_{x}^{(e)}$ (for $0 \leq e \in \mathbb{Z}$ ). The subgroup $G_{x}^{(e)}$ depends only on the cell containing $x$. If $x \leq y$, i.e., the cell containing $x$ is contained in the closure of the cell containing $y$, then $G_{x}^{(e)} \subset G_{y}^{(e)}$ (see [SS97, Proposition I.2.11]).

Define a $G$-equivariant cellular sheaf $\mathcal{S}^{(e)}$ on $X$ by putting $\mathcal{S}_{x}^{(e)}=V^{G_{x}^{(e)}}$; these stalks are finite-dimensional, since finite-length smooth representations are admissible. The restriction maps for $\mathcal{S}^{(e)}$ are defined by projecting $G_{x}^{(e)}$-fixed vectors onto $G_{y}^{(e)}$-fixed vectors, when $x \leq y$. In [SS97, Theorem IV.4.17], Schneider and Stuhler prove the following theorem.

Theorem 2.5. Suppose that $V=\operatorname{Ind}_{P}^{G}(E)$ for some $F$-parabolic subgroup $\mathbf{P}=$ $\mathbf{L U} \subset \mathbf{G}$, and irreducible supercuspidal representation $E$ of $L$. Then, for e suffciently large, $H_{c}^{\bullet}\left(X, \mathcal{S}^{(e)}\right)$ is nonzero in a single degree $d$, where it is isomorphic to $\operatorname{Ind}_{P}^{G}\left({ }^{n} E\right)$ as a representation of $G$. Here $n$ is a certain element of $N_{G}(L)$ and the degree $d$ lies between $0($ for $P=G)$ and $\operatorname{Rank}_{F}\left(\mathbf{G}_{\mathrm{der}}\right)$ (for $P$ minimal).

In particular, when $(\pi, V)$ is an irreducible supercuspidal representation of $G$,

$$
H_{c}^{0}\left(X, \mathcal{S}^{(e)}\right) \cong V \text { for } e \gg 0 .
$$

Corollary 2.6. Let $\mathbf{G}$ be a reductive group over a nonarchimedean local field $F$, whose derived subgroup has relative rank one. Let $G=\mathbf{G}(F)$. Then every irreducible supercuspidal representation of $G$ on a complex vector space is isomorphic to $\mathrm{cInd}_{K}^{G} \sigma$ for some compact-mod-center open subgroup $K \subset G$ and some irreducible representation $\sigma$ of $K$. 
Proof. Since $\mathbf{G}_{\text {der }}$ has relative rank one, the Bruhat-Tits building $X$ is a locally finite tree. Let $(\pi, V)$ be an irreducible supercuspidal representation of $G$. By Theorem 2.5, there exists a $G$-equivariant sheaf $\mathcal{S}$ on $X$, such that $H_{c}^{0}(X, \mathcal{S}) \equiv V$. Admissibility implies that $\operatorname{dim}\left(\mathcal{S}_{v}\right)<\infty$ for all $v \in V$. Since $G \backslash V$ is finite, $\operatorname{Rank}^{0}(\mathcal{S})$ is finite. Theorem 2.4 applies and proves the result.

Note that Vignéras Vig97, Theorem 4.6] has proven that the main results of Schneider and Stuhler adapt to representations of $G$ on $R$-vector spaces, when $R$ is a field whose characteristic is coprime to the residue characteristic of $F$. Hence the previous result holds for cuspidal (see [Vig97, §3.7] for the definition) $R$-representations as well as for supercuspidal complex representations.

\section{ACKNOWLEDGMENTS}

The author thanks Gordan Savin, Stephen DeBacker, Jeff Adler, Jeffrey Hakim, and Jessica Fintzen for helpful discussions about representations of $p$-adic groups, buildings, and $K$-types. The author also thanks Paul Broussous, who looked at an earlier version of the manuscript and made helpful suggestions including a strengthening of the main theorem. The author thanks the anonymous referees, who pointed out the previous work of Ol'shanskil and whose suggestions strengthened the exposition.

\section{REFERENCES}

[BK93] Colin J. Bushnell and Philip C. Kutzko, The admissible dual of GL(N) via compact open subgroups, Annals of Mathematics Studies, vol. 129, Princeton University Press, Princeton, NJ, 1993. MR1204652

[Car] Lisa Carbone, On the classification of rank 1 groups over non-archimedean local fields, Lecture notes from a $p$-adic groups seminar at Harvard University.

[Kim07] Ju-Lee Kim, Supercuspidal representations: an exhaustion theorem, J. Amer. Math. Soc. 20 (2007), no. 2, 273-320, DOI 10.1090/S0894-0347-06-00544-3. MR.2276772

[Ol'77] G. I. Ol'šanskiü, Classification of the irreducible representations of the automorphism groups of Bruhat-Tits trees (Russian), Funkcional. Anal. i Priložen. 11 (1977), no. 1, 32-42, 96. MR0578650

[SS97] Peter Schneider and Ulrich Stuhler, Representation theory and sheaves on the BruhatTits building, Inst. Hautes Études Sci. Publ. Math. 85 (1997), 97-191. MR.1471867

[Ste08] Shaun Stevens, The supercuspidal representations of p-adic classical groups, Invent. Math. 172 (2008), no. 2, 289-352, DOI 10.1007/s00222-007-0099-1. MR2390287

[Tit79] J. Tits, Reductive groups over local fields, Automorphic forms, representations and $L$ functions (Proc. Sympos. Pure Math., Oregon State Univ., Corvallis, Ore., 1977), Proc. Sympos. Pure Math., XXXIII, Amer. Math. Soc., Providence, R.I., 1979, pp. 29-69. MR.546588

[Vig97] Marie-France Vignéras, Cohomology of sheaves on the building and R-representations, Invent. Math. 127 (1997), no. 2, 349-373, DOI 10.1007/s002220050124. MR.1427623

[Yu01] Jiu-Kang Yu, Construction of tame supercuspidal representations, J. Amer. Math. Soc. 14 (2001), no. 3, 579-622, DOI 10.1090/S0894-0347-01-00363-0. MR.1824988

Department of Mathematics, University of California, Santa Cruz, California 95064 Email address: weissman@ucsc.edu 